With regard to treatment gentle curetting of the anterior ethmoidal area through the nose was followed by an escape of pus. Hydrogen peroxide was instilled often into the left nostril. Fomentations were applied to the left eye.

On July 11 the proptosis was much reduced. On July 16 the proptosis had disappeared. There was no abnormal orbital resistance. The fundus was normal. Discharge from the left nostril was still copious. On August 8, the child was discharged as cured.

\title{
COUNCIL OF BRITISH OPHTHALMOLOGISTS
}

\section{Report on the desirability of a special qualification in ophthalmology}

THIS report is divided into five parts :

I.-The existing conditions concerning examinations in special subjects.

II.-Examinations in Ophthalmology in the United Kingdom.

III.-The qualifications required by the principal hospitals of the United Kingdom for appointment to the post of Ophthalmic Surgeon.

IV.-The desirability of a special examination in Ophthalmology. V.-Conclusions and recommendations.

\section{I.-The existing conditions concerning examinations in special subjects}

At present there are in the United Kingdom special examinations in Dental Surgery, Public Health, Tropical Medicine and Ophthalmology.

These examinations may be classified in two groups:

(a) Those in which the special subjects form part of a higher examination in medicine or surgery, e.g., Tropical Medicine; State Medicine; Ophthalmic Surgery; Aural, Nasal and Laryngeal Surgery; and Dental Surgery for the M.D. or M.S. of the University of London: Ophthalmology; Aural, Nasal and Laryngeal Surgery; Advanced Midwifery with Obstetric Surgery and Gynaecology; Advanced Anatomy; and Dental Surgery for the Fellowship of the Royal College of Surgeons, Edinburgh.

(b) Those in which qualified medical men are examined in a special subject, independently of any examination in general medicine or surgery, e.g., for the Diploma in Public Health, University of Cambridge; the Licence in Dental Surgery, Royal College of Surgeons, England; and the Diploma in Ophthalmology, University of Oxford. 


\section{II.-Examinations in Ophthalmology in the United Kingdom}

The first examination in Ophthalmology as a special subject was established by the Royal College of Surgeons of Edinburgh, in 1883. In the examination for the Fellowship, Ophthalmology is included in the list of special subjects, of which one must be taken. The diploma granted bears no indication of the special subject in which the candidate was examined.

At the University of London, Ophthalmology has recently been recognised as a special branch of the M.S. degree under the following conditions :-

Every candidate for the degree of Master of Surgery must have taken the degree of Bachelor of Medicine and Bachelor of Surgery in this University not less than two years previously, except as provided below.

Candidates who have either (I) the M.B., B.S. degrees witk Honours in Surgery ; or (II) subsequently to taking the M.B., B.S. degrees, conducted a piece of oríginal work approved by the University, or (III) subsequently to taking the M.B., B.S. degrees had such exceptional experience as may be approved for this purpose by the University, may present themselves after an interval of one year only from the time of taking the M.B.. B.S. degrees provided that they comply in other respects with the conditions stated in the following paragraphs:

Certificates.-Every candidate must forward together with his form of entry the following certificates:-

1. A certificate of having spent at least two years in the study and practice of ophthalmology at a teaching school or schools approved by the University, of which one year at least must have been subsequent to obtaining the M.B., B.S. degrees in this University.

2. A certificate of having held during this time for at least six months an approved appointment in the ophthalmic department of a general hospital recognised by the University for the purpose, or at an ophthalmic hospital recognized by the University, Details of examination. - The examination will consist of :-

Two papers in ophthalmology (in one of which there may be included a case for commentary.)

One paper in the principles of surgery.

A clinical examination.

One paper in the anatomy and physiology of the eye and other parts concerned in ophthalmology (including physiological optics).

One paper in the pathology of the eye and other parts concerned in ophthalmology. An oral examination.

Candidates shall not be approved by the examiners unless they have shown a competent knowledge in all the subjects of the examination.

At the University of Oxford a diploma in ophthalmology (D.O.,Oxon), was instituted in 1910. It is granted under the following conditions :-

Candidates must have their names on the medical register of the United Kingdom, unless, being Bachelors of Medicine or Doctors of Medicine of universities outside the United Kingdom, they have obtained special permission from the Board of the Faculty of Medicine.

Candidates shall be required, at the times of entering their names, to produce the following certificates:-

A certificate or certificates showing that the candidate has duly attended a course of clinical ophthalmology for twelve calendar months in connection with hospitals or institutions recognised for the purpose by the Board of the Faculty of Medicine.

A certificate showing that the candidate has satisfactorily carried out in the University of Oxford the practical study of the anatomy of the eye and its appendages, including the development and minute structure of these parts. 
A certificate showing that the candidate has satisfactorily carried out in the University of Oxford the practical study of physiological optics and the physiology of vision.

A certificate showing that the candidate has duly attended a course of ophthalmology in the University of Oxford for a period of at leasst two months.

The subjects of the examination are dioptrics, diseases of the eye, ophthalmology in general medicine, clinical cases and ophthalmic surgery.

In the University of Liverpool a diploma in ophthalmology was instituted, but is not now granted.

\section{Examinations in Ophthalmology in America}

\section{University of Colorado.}

Degree of Doctor of Ophthalmology inaugurated in 1913.

Requirements for the Degree. The degree of Doctor of Ophthalmology will be granted to those meeting the following requirements :-

A. Evidence of graduation at a standard medical school, and of a preliminary education covering high school algebra, geometry, plane trigonometry, and elementary optics.

B. One year of clinical work of 300 days, two hours per day :-

1. By service as interne in a hospital for diseases of the eye :

2. By service as clinical assistant in an approved out-patient department for the treatment of eye diseases :

3. By regular attendance in a school for post-graduate medical teaching, where the students are given sufficient facilities for the actual examination and treatment of patients :

4. By serving as assistant to an ophthalmologist in private practice, who is recognised as a competent teacher of clinical ophthalmology :

5. Or by a combination of the above :

C. One or more summer courses in ophthalmology at the University of Colorado.

D. Each Candidate must pass an examination, written, oral, microscopical, and clinical ; and must submit an original thesis, and stand an examination thereon.

The course is planned to fill the time of two scholastic years and consists of optics, histology, pathology, ophthalmology, and operative surgery.

University of Minnesota.

Doctor of Ophthalmology. In this University a course similar to that in the University of Colorado has been instituted, but all the work is expected to be done in the University.

\section{III - The qualifications required by the principal hospitals of the United Kingdom of candidates for the post of ophthalmic surgeon}

A circular letter was sent to all the principal hospitals in the United Kingdom asking what professional qualifications were required of candidates for the post of ophthalmic surgeon.

Replies were obtained showing that 17 required the Fellowship of the Royal College of Surgeons, England; 5 the Fellowship of the Royal College of Surgeons, Edinburgh; 4 the Fellowship of the Royal College of Surgeons, Ireland ; 2 the degree of Master of Surgery of a University or the Fellowship of the Royal College of 
Surgeons, England; 8 a medical degree of a University or the Fellowship of one of the Royal Colleges of Surgeons of the United Kingdom. (Three of the last group will accept the Membership of the Royal College of Surgeons of England.) Eighteen do not require any special qualification.

.The following five hospitals require evidence of special training in ophthalmology:-Belfast Ophthalmic Hospital, two years; Central London Ophthalmic Hospital, two years; Royal Westminster Ophthalmic Hospital, two years; Western Ophthalmic Hospital, two years; Liverpool Eye and Ear Hospital, six months.

Under present conditions the degrees or diplomas which candidates for the posts of ophthalmic surgeon to the principal hospitals in the United Kingdom must hold, while they testify to proficiency in general medicine and surgery, furnish no evidence of special knowledge of ophthalmology.

\section{IV.-The desirability of a special examination in ophthalmology}

In the higher surgical examinations as at present conducted there is seldom any adequate test of a candidate's knowledge of ophthalmology. The examiners are general surgeons who are not thoroughly conversant with the subject, and there is, therefore, a natural tendency on the part of the student to devote insufficient attention to this branch of surgery. Formerly the examining boards included a proportion of surgeons who practised as ophthalmologists, and in consequence the subject was more frequently and adequately dealt with.

The importance of ophthalmic knowledge and experience in relation to the welfare of the population is now widely recognized. The large and increasing number of appointments for which such knowledge and experience are required renders it essential that candidates for these posts should be able to show, not only that they have gone through a satisfactory course of training in ophthalmology, but that they have attained a certain standard of proficiency, as tested by examination.

The practice of ophthalmology must be based upon an accurate knowledge of the anatomy and physiology of the visual organs, optics, and the pathology of the eye. The establishment of a special examination in ophthalmology would ensure that candidates have been adequately trained in and possess a sufficient knowledge of these subjects. It would at the same time be an incentive to the special hospitals to provide increased facilities for instruction.

The close relationship of ophthalmic to general surgery renders it imperative that those who propose to practise as ophthalmic surgeons should possess a sound knowledge of the general principles of surgery, pathology, bacteriology, etc., and of their application to the surgery of the eye, orbit, and surrounding structures. The 
Council therefore hold that an examination in ophthalmology should form part of the examination for a higher degree or diploma in surgery, as is now the case at the University of London and the Royal College of Surgeons, Edinburgh, rather than that there should be a special examination in ophthalmology alone.

If such an examination is adopted it is desirable that it should be definitely stated that the degree or diploma is granted in ophthalmology.

The Council are of opinion that every candidate before presenting himself for examination should be required to produce evidence that he has studied ophthalmology during a period of two years, of which one has been spent in holding recognized ophthalmic appointments.

\section{V.-Conclusions and Recommendations}

(1) It is desirable that the universities and colleges in the United Kingdom should make provision for a special examination in ophthalmology for those who propose to devote themselves to this branch of medicine.

(2) It is desirable that ophthalmology should be one of the optional subjects in which a candidate can elect to be examined for the degree of Master of Surgery of a.University, or for the diploma of Fellow of a Royal College of Surgeons, as is at present the case at the University of London and the Royal College of Surgeons, Edinburgh.

(3) Before presenting himself for such an examination the candidate should furnish evidence of at least two years' study of ophthalmology, one of which has been spent in holding recognized ophthalmic appointments.

(4) The special examination in ophthalmology should be written, oral, and practical, and should comprise the following subjects: anatomy, pathology, optics, systematic and clinical ophthalmology, and operative surgery.

\section{ANNOTATIONS}

\section{Illuminating Engineering Society}

A recent number of the Illuminating Engineer, the official journal of the Illuminating Engineering Society, gives a summary of the papers read before the Society since its foundation in 1909. The record is a very satisfactory one. During the war, committees of the Society did most useful work, some of which we have referred to in a previous number (p. 207, 1919). The more intimate relation 